RIKEN-TH-190

\title{
Uniformization, Calogero-Moser/Heun duality and Sutherland/bubbling pants
}

\author{
Ta-Sheng Ta:* \\ Theoretical Physics Laboratory, RIKEN, Wako, Saitama 351-0198, JAPAN
}

\begin{abstract}
Inspired by the work of Alday, Gaiotto and Tachikawa (AGT), we saw the revival of Poincaré's uniformization problem and Fuchsian equations obtained thereof.

Three distinguished aspects are possessed by Fuchsian equations. First, they are available via imposing a classical Liouville limit on level-two null-vector conditions. Second, they fall into some $A_{1}$-type integrable systems. Third, the stress-tensor present there (in terms of the $Q$-form) manifests itself as a kind of one-dimensional "curve".

Thereby, a contact with the recently proposed Nekrasov-Shatashvili limit was soon made on the one hand, whilst the seemingly mysterious derivation of Seiberg-Witten prepotentials from integrable models become resolved on the other hand. Moreover, AGT conjecture can just be regarded as a quantum version of the previous Poincaré's approach.

Equipped with these observations, we examined relations between spheric and toric (classical) conformal blocks via Calogero-Moser/Heun duality. Besides, as Sutherland model is also obtainable from Calogero-Moser by pinching tori at one point, we tried to understand its eigenstates from the viewpoint of toric diagrams with possibly many surface operators (toric branes) inserted. A picture called "bubbling pants" then emerged and reproduced well-known results of the non-critical self-dual $c=1$ string theory under a "blown-down" limit.
\end{abstract}

\section{Introduction}

Recently, AGT conjecture [1] has launched extremely active investigations towards both 2D Liouville CFT and $4 \mathrm{D} \mathcal{N}=2 S U(2) \mathrm{SCFT}$ or even generalization, say, higher rank of gauge groups [2], non-conformal (asymptotically-free) limit [3, 4] and $q$-deformation [5, 6] to name a few. Now, it seems rather appropriate to consider not only proving (or checking) this conjecture but also why these two apparently irrelevant arenas should coincide with each other.

In this short article, we observe that the "uniformization problem" pioneered by Klein, Koebe and Poincaré more than a century ago may shed illuminating light on answering this question 1 . For instance, the stress-tensor $T(z)$ showing up there turns out to provide the $S U(2)$ Gaiotto (rewritten Seiberg-Witten) curve [9] under certain proper limit, say, large intermediate $s$-channel momentum. Consequently, AGT conjecture strongly manifests itself as a full quantum uplift of the uniformization problem w.r.t. two equivariant parameters $\left(\epsilon_{1}, \epsilon_{2}\right)$. In addition, Fuchsian type differential equations 2

$$
\left(\partial_{z}^{2}+T(z)\right) \Psi=0
$$

\footnotetext{
* e-mail address: tasheng@riken.jp

${ }^{1}$ See [7, 8] for issues about uniformizing four-punctured Riemann spheres

${ }^{2}$ Second-order differential equations are of Fuchsian type if they are fixed by certain Riemann scheme and meanwhile all their singularities are regular.
} 
which are present during uniformizing punctured Riemann surfaces are as well available through the (semi)classical Liouville limit $b \rightarrow 0$ (or infinitely large central charge $c \rightarrow \infty$ ) imposed onto the constraint for a null vector at the second level in Verma module, i.e.

$$
\left(L_{-2}-\frac{3}{2\left(2 \Delta\left(h_{2,1}\right)+1\right)} L_{-1}^{2}\right) \Phi_{2,1}=0, \quad h_{r, s}=\frac{1-r}{2} b+\frac{1-s}{2 b}, \quad h_{2,1}=-\frac{b}{2} .
$$

As a matter of fact, this classical limit can get readily identified with the so-called NekrasovShatashvili (NS) limit [10] ( $g_{s}$ : string coupling)

$$
\epsilon_{1}=b g_{s} \rightarrow 0, \quad \epsilon_{2}=\frac{g_{s}}{b}=\text { fixed }, \quad g_{s} \rightarrow 0, \quad b \rightarrow 0
$$

which ultimately has something to do with many well-known integrable systems. Performing the WKB method onto the equation (1.1) satisfied by $\mathcal{N}=2$ instanton partition functions with one surface operator inserted under NS limit, one is able to obtain either classical conformal blocks [11] $]^{3}$ or $\log Z_{\text {inst }}\left(\epsilon_{1}, \epsilon_{2}=0\right)$ following AGT who claimed a much more general relationship

$$
Z_{\text {inst }}\left(\epsilon_{1}, \epsilon_{2}\right)=\mathcal{B}\left(b, \frac{1}{b}\right)
$$

Many publications [13, 14, 15, 16, 17, 18, 19, 20, 21] have been devoted into this direction.

In summary, the philosophy we are after is as follows. Because every $\mathcal{N}=2 A_{1}$-type SCFT is associated with some genus- $g$-punctured Riemann surface $C_{g, n}[9]$, through uniformizing $C_{g, n}$ both a classical piece of Liouville theory and an integrable (Fuchsian) system will be further inferred. This viewpoint therefore renders us the clue for understanding why these two seemingly different arenas ultimately meet.

Based on these, we start off to consider a four-punctured Riemann sphere $\mathbb{P}^{1} \backslash\left\{z_{1}, \cdots, z_{4}\right\}$ over which a second-order Heun equation is defined. Obviously, it can be thought of as an extension of usual hypergeometric differential equations living on $\mathbb{C} \backslash\{0,1\}$ fixed by certain Riemann scheme 4 . It then seems plausible that classical spheric five-point conformal blocks having one null vector

Table 1: Riemann scheme in the presence of five regular singular points on $\mathbb{P}^{1} . \chi^{\text {'s }}$ are called characteristic exponents.

\begin{tabular}{|c|c|c|c|c|}
\hline$z=0$ & $z=1$ & $z=t$ & $z=\lambda$ & $z=\infty$ \\
\hline 0 & 0 & 0 & 0 & $\chi_{1}$ \\
\hline$\chi_{2}$ & $\chi_{3}$ & $\chi_{4}$ & $\chi_{5}$ & $\chi_{6}$ \\
\hline
\end{tabular}

$\Phi_{2,1}$ insertion at the second level satisfy Heun equations. We clarify this observation via a celebrated mathematical duality: Heun can be suitably transformed into a two-body $\left(A_{1}\right.$-type) elliptic Calogero-Moser (eCM) model. Finally, in view of a marvelous limit bringing eCM to Sutherland (trigonometric Calogero-Sutherland) model, we develop some correspondence between its excited wave functions and the toric diagram geometrically engineering $\mathcal{T}_{0,3}\left(A_{1}\right)$ theory.

We organize this article as follows. In section 2, we explicitly re-derive the $2 N_{c}=N_{f}=4$ SW prepotential upon taking into account two approaches: hermitian matrix model and classical

\footnotetext{
${ }^{3}$ Their quantum versions are called Belavin-Polyakov-Zamolodchikov (BPZ) conformal blocks $\mathcal{B}[12$. See the definition around (2.7).

${ }^{4}$ See [22] for an interpretation of triality in the $S U(2)$ Seiberg-Witten theory in terms of Gauss hypergeometric functions.
} 
Liouville theory. One may think of this presentation as another derivation other than the original AGT proposal. This is because we have just relied on the Ward identity of $T(z)$ in the uniformization problem and arguments intrinsic to hermitian matrix models. In section 3 , we proceed to review Heun/eCM duality and study the relationship between toric and spheric conformal blocks (elliptic/non-elliptic $\mathcal{N}=2 S U(2) \times S U(2)$ SCFTs). Also, we find new insights into eigenstates of Sutherland model from the toric diagram perspective. Finally, a chart summarizing the main idea is pasted in section 4 .

\section{Uniformization problem}

Owing to Klein, Koebe and Poincaré, one is capable of uniformizing a punctured Riemann surface by means of hyperbolic geometry. For instance, there exists uniquely a hyperbolic metric

$$
d s^{2}=e^{\varphi(z, \bar{z})} d z d \bar{z}
$$

on an $n$-punctured Riemann sphere $X=\mathbb{P}^{1} \backslash\left\{z_{1}, \ldots, z_{n}\right\}$ which has the constant negative curvature $R=-8 \pi \mu b^{2}$ and is obviously in the same conformal class as the flat space $d s^{2}=d z d \bar{z}$. One is as well able to think of (2.1) as the pull-back from Poincaré's hyperbolic metric of a unit disc $\mathbf{D}$ (or upper half-plane $\mathbb{H}) \sqrt[5]{5}$, i.e.

$$
d s^{2}=e^{\varphi(z, \bar{z})} d z d \bar{z}=\frac{4\left|\eta^{\prime}\right|^{2}}{\left(1-|\eta|^{2}\right)^{2}} d z d \bar{z}, \quad \eta(z): \text { uniformization map. }
$$

Now, the requirement $R=-8 \pi \mu b^{2}$ leads to the familiar Liouville equation

$$
\partial_{z} \partial_{\bar{z}} \varphi=2 \pi \mu b^{2} e^{\varphi}
$$

which is solved by $\varphi_{c l}$, the stationary point of some Liouville functional $\mathcal{S}[\varphi]$ being clarified later. Note that our conventions are listed below

$$
Q=b+1 / b, \quad b: \text { dimensionless Liouville coupling constant, } \quad \mu: \text { cosmological constant. }
$$

Of course, the asymptotic behavior of $\varphi_{c l}$ around $z_{i}(i=1, \ldots, n)$ is readily read off according to the weight assigned to them. A guideline laid down by Polyakov to which we will adhere is that a path integral over $\phi$, say,

$$
\int \mathcal{D} \phi \prod_{i=1}^{n} V_{i}\left(z_{i}\right) \exp (\mathcal{S}[\phi])
$$

with $V_{i}=\exp \left(2 \alpha_{i} \phi\right)$ whose conformal dimension is $\Delta_{i}=\alpha_{i}\left(Q-\alpha_{i}\right)$, should be equivalent to $\int \mathcal{D} \phi \exp (\mathcal{S}[\phi])$ where instead $\phi$ is endowed with singularities at $z_{i}$ 's and vertex operators become absent thereof. Set $\varphi=2 b \phi$ and $S[\phi]=\frac{1}{b^{2}} \mathcal{S}[\varphi]$ in order to facilitate a classical analysis through $b \rightarrow 0$. Here, $S(\phi)$ stands for the bare Liouville functional (field).

Next, construct from $\varphi_{c l}$ a standard Liouville $(2,0)$ stress-tensor $T_{L}(z)=Q \partial_{z}^{2} \phi-\left(\partial_{z} \phi\right)^{2}$. Ac-

\footnotetext{
${ }^{5}$ While what is isomorphic to $X$ is often $\mathbf{D} / \Gamma$ with $\Gamma \subset P S U(1,1)$ being the isometry group of $\mathbf{D}$, it is harmless to directly make use of the metric of $\mathbf{D}$ in this manner.
} 
cording to Poincaré one has the following expansion (when all punctures are parabolic):

$$
T(z) \equiv \frac{1}{2} \partial_{z}^{2} \varphi_{c l}-\frac{1}{4}\left(\partial_{z} \varphi_{c l}\right)^{2}=\sum_{i=1}^{n-1} \frac{1}{4\left(z-z_{i}\right)^{2}}+\frac{c_{i}}{\left(z-z_{i}\right)}
$$

where accessory parameters $c_{i}$ 's satisfy three linear constraints yielded by imposing

$$
T(z) \rightarrow \frac{1}{4 z^{2}}+\frac{c_{n}}{z^{3}}+\mathcal{O}\left(\frac{1}{z^{4}}\right), \quad z \rightarrow \infty .
$$

We will always assume $\left(z_{1}, z_{n-1}, z_{n}\right)=(0,1, \infty)$ under a suitable Möbius transformation. Note that accessory parameters can get either determined by monodromies of (1.1) on $\mathbb{P}^{1}$ or the so-called Polyakov conjecture upon exploiting the Ward identity w.r.t. $T_{L}(z)$ :

$$
c_{i}=-\frac{\partial \mathcal{S}\left[\varphi_{c l}\left(\delta_{i}, z_{i}\right)\right]}{\partial z_{i}}, \quad i \neq(0,1, \infty)
$$

In contrast to $\Delta_{i}$, here $\delta_{i}=\xi_{i}\left(1-\xi_{i}\right)=\frac{1}{4}\left(1-\mu_{i}^{2}\right)$ denotes the classical conformal weight of inserted vertex operators. Besides, each zero (non-zero) $\mu_{i}$ corresponds to a parabolic (elliptic) puncture. Remarkably, (2.4) has been proved rigorously by Zograf and Takhtajan [23] when all punctures are parabolic ones.

\subsection{Fuchsian differential equation}

Recall that the $Q$-form of Fuchsian equations looks like

$$
\partial_{z}^{2} y+\frac{1}{2}\{\rho, z\} y=0, \quad T(z)=\frac{1}{2}\{\rho, z\}: \text { Schwarzian derivative of } \rho, \quad \rho=\frac{y_{\vartheta}}{y_{\varsigma}} .
$$

(2.5) is fixed by both the number of regular singularities placed on $\mathbb{C}$ and a prescribed Riemann scheme. Here, the multivaluedness of $\rho$ is accounted for due to its form being a ratio of two independent solutions to (2.5). Also, the pair $\left(y_{\vartheta}, y_{\varsigma}\right)$ may get normalized to have a unit Wronskian:

$$
\left(\partial_{z} y_{\vartheta} \cdot y_{\varsigma}-y_{\vartheta} \cdot \partial_{z} y_{\varsigma}\right)=1
$$

which helps fix the conjugation acquired from $S L(2, \mathbb{C})$. In view of (2.5), a type of Riemann-Hilbert $(\mathrm{RH})$ problem can be raised. Namely, once monodromies of $\rho$ in $S U(1,1)$ are found explicitly, all $c_{i}$ 's get known subsequently. This sounds like the usual RH problem only when the latter statement is replaced by "there exists certain algebraic curve whose period integrals reproduce $\left(y_{\vartheta}, y_{\varsigma}\right)$ ".

The stress-tensor $T(z)$ thus obtained by computing Schwarzian is a meromorphic function on $\mathbb{P}^{1}$ :

$$
T(z)=\sum_{i=1}^{n-1} \frac{\delta_{i}}{\left(z-z_{i}\right)^{2}}+\frac{c_{i}}{\left(z-z_{i}\right)}
$$

As before, its asymptotic behavior at infinity is supposed to be regular such that

$$
T(z) \rightarrow \frac{\delta_{n}}{z^{2}}+\frac{c_{n}}{z^{3}}+\mathcal{O}\left(\frac{1}{z^{4}}\right)
$$


is able to impose three linear equations on $c_{i}$ 's:

$$
\sum_{i=1}^{n-1} c_{i}=0, \quad \sum_{i=1}^{n-1}\left(\delta_{i}+c_{i} z_{i}\right)=\delta_{n}, \quad \sum_{i=1}^{n-1}\left(2 \delta_{i} z_{i}+c_{i} z_{i}^{2}\right)=c_{n} .
$$

As a remark, according to

$$
T(z)=\frac{1}{2}\{\rho(z), z\}=\frac{2 \partial^{3} \rho \cdot \partial \rho-3\left(\partial^{2} \rho\right)^{2}}{4(\partial \rho)^{2}}, \quad \partial \equiv \partial_{z},
$$

an equivalent description of this expression goes back precisely to (2.2) if one equates $\eta$ with $\rho$.

Let us illustrate more concretely what was announced around (1.2). That is, define

$$
\left\langle\Phi_{2,1}(z)\right\rangle \equiv \Psi(z)=\left\langle V_{4}(\infty) V_{3}(1) \Phi_{2,1}(z) V_{2}(x) V_{1}(0)\right\rangle
$$

at the level of BPZ conformal blocks. (1.2) lays down $\left(\Delta=h_{2,1}\right)$

$$
\begin{aligned}
& {\left[\frac{\partial^{2}}{\partial z^{2}}+\gamma\left(\frac{1}{z}-\frac{1}{1-z}\right) \frac{\partial}{\partial z}-\gamma\left(\frac{\Delta_{1}}{z^{2}}+\frac{\Delta_{2}}{(z-x)^{2}}+\frac{\Delta_{3}}{(1-z)^{2}}+\frac{\Lambda}{z(1-z)}+\frac{x(1-x)}{z(z-x)(1-z)} \frac{\partial}{\partial x}\right)\right]} \\
& \times\left\langle\Phi_{2,1}(z)\right\rangle=0
\end{aligned}
$$

for the null-vector where

$$
\Lambda=\Delta_{1}+\Delta_{2}+\Delta_{3}+\Delta-\Delta_{4}, \quad \gamma=\frac{2}{3}(2 \Delta+1) .
$$

By $b \rightarrow 0$, the above equation becomes Fuchsian:

$$
\left(\partial_{z}^{2}+T(z)\right) \Psi^{c l}(z)=0
$$

where

$$
T(z)=\frac{\delta_{1}}{z^{2}}+\frac{\delta_{2}}{(z-x)^{2}}+\frac{\delta_{3}}{(1-z)^{2}}+\frac{\delta_{1}+\delta_{2}+\delta_{3}-\delta_{4}}{z(1-z)}+\frac{x(1-x) c(x)}{z(z-x)(1-z)} .
$$

$T(z)$ here does not look like (2.6) because $(0,1, \infty)$ have been chosen in order to eliminate constraints due to projective invariance. Note that the accessory parameter $c(x)$ will be given in (2.10) later on.

\subsection{Classical Liouville theory}

From now on, we mainly focus on $\mathbb{P}^{1} \backslash\{0, x, 1, \infty\}$ such that $z_{2}=x$ stands for the cross-ratio. While the classical limit $b \rightarrow 0$ is taken, an $n$-point function $\mathcal{G}\left(\alpha_{1}, \cdots, \alpha_{n}\right)$ computed w.r.t. $S[\phi]$ gets approximated by

$$
\mathcal{G}\left(\alpha_{1}, \cdots, \alpha_{n}\right) \sim \exp \left(-\frac{1}{b^{2}} \mathcal{S}\left[\varphi_{c l}\right]\right)=\exp \left(-\frac{1}{b^{2}} \mathcal{S}_{c l}\left[\xi_{1}, \cdots, \xi_{n} ; z_{1}, \cdots, z_{n}\right]\right)
$$

where $\varphi_{c l}$ again satisfies (2.2) as well as all required asymptotics with $\alpha_{i}=\xi_{i} / b$ being very heavy. Moreover, as $Q^{2} \rightarrow 1 / b^{2}$ the quantum BPZ conformal block becomes

$$
\mathcal{F}_{\Delta}\left[\begin{array}{cc}
\Delta_{3} & \Delta_{2} \\
\Delta_{4} & \Delta_{1}
\end{array}\right](x) \sim \exp \left(Q^{2} f_{\delta}\left[\begin{array}{ll}
\delta_{3} & \delta_{2} \\
\delta_{4} & \delta_{1}
\end{array}\right](x)\right), \quad \delta=\frac{1}{4}+p^{2}
$$


where $f$ represents the classical conformal block [11. Also, since $Q^{2} \rightarrow \infty$ the intermediate momentum $p$ is forced to be $p_{s}, s$-channel saddle point momentum, which solves $\left(\delta_{s} \equiv \frac{1}{4}+p_{s}^{2}\right)$

$$
\left.\frac{\partial \mathcal{S}\left[\xi_{1}, \cdots, \xi_{4} ; \delta ; x\right]}{\partial p}\right|_{p=p_{s}}=0, \quad \mathcal{S}\left[\xi_{1}, \cdots, \xi_{4} ; \delta_{s} ; x\right]=\mathcal{S}_{c l}\left[\xi_{1}, \cdots, \xi_{4} ; x\right] .
$$

Needless to say, $\mathcal{F}_{\Delta}\left(\Delta_{i}, x\right)$ plays a central rule in the full Liouville four-point function 6 :

$$
\mathcal{G}\left(\alpha_{1}, \cdots, \alpha_{4} ; x, \bar{x}\right)=\frac{1}{2} \int_{-\infty}^{\infty} d P C\left(\alpha_{1}, \alpha_{2}, \frac{Q}{2}+i P\right) \cdot C\left(\alpha_{3}, \alpha_{4}, \frac{Q}{2}-i P\right) \cdot\left|\mathcal{F}_{\Delta}\left(\Delta_{i} ; x\right)\right|^{2}
$$

where $C$ is recognized as the structure constant and $P=p / b\left(P^{2}=\Delta-\frac{1}{4} Q^{2}\right)$.

What comes as a classical counterpart of (2.8) is

$$
\mathcal{S}_{c l}\left[\xi_{1}, \cdots, \xi_{4} ; x\right]=\mathcal{S}^{(3)}\left(\delta_{4}, \delta_{3}, \delta_{s}\right)+\mathcal{S}^{(3)}\left(\delta_{s}, \delta_{2}, \delta_{1}\right)-f_{\delta_{s}, \delta_{i}}(x)-f_{\delta_{s}, \delta_{i}}(\bar{x})
$$

What was referred to as Polyakov conjecture now states that

$$
c_{2}(x)=-\frac{\partial \mathcal{S}_{c l}\left[\xi_{i} ; x\right]}{\partial x}=\left(\frac{\partial}{\partial x} f_{\delta}\left[\begin{array}{ll}
\delta_{3} & \delta_{2} \\
\delta_{4} & \delta_{1}
\end{array}\right](x)\right)_{p=p_{s}(x)}
$$

where the second equality is derived by taking into account (2.9). Notice that $\ell\left(\gamma_{12}\right)=4 \pi p_{s}$ where $\gamma_{12} \equiv \gamma(x)$ represents the geodesic length of a (hyperbolic) four-punctured Riemann sphere and encircles two punctures $\left(z_{1}, z_{2}\right) \equiv(0, x)$ from other ones.

\subsection{Hermitian matrix model}

Because the relationship amongst (2.10) is entirely chiral and stems from the familiar Ward identity about $T_{L}(z)$ inserted at the level of conformal blocks, it is tempting to compare (2.10) with what takes place in a hermitian matrix model possessing an usual Vandermonde7. That is, by means of Kodaira-Spencer field (chiral free boson) $\phi_{K S}$, the expectation value of the matrix model stresstensor $T_{M}(z)$ given by

$$
\begin{aligned}
& y^{2}=\left\langle T_{M}(z)\right\rangle \rightarrow \mathcal{W}^{\prime}(z)^{2}+f(z), \quad \text { when } \hbar=1 / N \rightarrow 0, \\
& T_{M}(z)=\sum_{n} L_{n} z^{-n-2}=\left(\partial \phi_{K S}\right)^{2}, \\
& Z=\frac{1}{\operatorname{vol} U(N)} \int_{N \times N} d M \exp \left(\frac{1}{\hbar} \operatorname{Tr} \mathcal{W}(M)\right)=\exp \left(\sum_{g \geq 0} \hbar^{2 g-2} \mathcal{F}_{g}\right), \\
& \left\langle\partial \phi_{K S}(z)\right\rangle=\mathcal{W}^{\prime}(z)+2 \hbar \operatorname{Tr} \log (z-M)=\left(t_{0} z^{-1}+\sum_{n>0} n t_{n} z^{n-1}+2 \hbar^{2} \sum_{n \geq 0} z^{-n-1} \frac{\partial}{\partial t_{n}}\right) Z
\end{aligned}
$$

leads to the large- $N$ spectral curve. Certainly, its Ward identity bears a strong resemblance to (2.10) but now $f$ has to be replaced by $\hbar^{-2} \mathcal{F}_{0}$ (with possibly $\phi_{K S} \rightarrow \hbar^{-1} \phi_{K S}$ as $\hbar \rightarrow 0$ ). $L_{n}$ 's are Virasoro generators realized by $\phi_{K S}$ possessing the central charge $c=1$.

We naturally anticipate that if the spectral curve $y^{2}=\lim _{\hbar \rightarrow 0}\left\langle T_{M}(z)\right\rangle$ is recognized as Gaiotto curve (rewritten Seiberg-Witten curve), $\mathcal{F}_{0}$ of $Z$ gets equivalent to the low-energy $\mathcal{N}=2 \mathrm{SW}$

\footnotetext{
${ }^{6}$ We have omitted the factor $\prod_{i<j}\left|x_{i j}\right|^{2 \gamma_{i j}}$ with $\gamma_{12}=\gamma_{13}=0, \gamma_{14}=-2 \Delta_{1}, \gamma_{24}=\Delta_{1}+\Delta_{3}-\Delta_{2}-\Delta_{4}, \gamma_{34}=$ $\Delta_{1}+\Delta_{2}-\Delta_{3}-\Delta_{4}$ and $\gamma_{23}=\Delta_{4}-\Delta_{1}-\Delta_{2}-\Delta_{3}$ inside $\mathcal{G}$.

${ }^{7}$ This section is fully inspired by (3.41) of Eguchi and Maruyoshi [24].
} 
prepotential. The concrete form of $Z$ has already been proposed by Dijkgraaf and Vafa [25] last year (see [26, 27, 28] for further refinements). Below, we will see that $f_{\delta_{s}, \delta_{i}}(x)$ of (2.7) turns out to give us the desired infra-red prepotential $\mathcal{F}^{S W}$.

\subsection{From null-state condition to Fuchsian (Schrödinger-like) equation}

Another distinguished aspect we want to review involves a degenerate field $V_{-\frac{b}{2}}$ entering the standard Liouville theory. Due to the null-vector decoupling equation at the second level $L_{-1}^{2}+b^{2} L_{-2}=$ 0 , conformal blocks involving $V_{-\frac{b}{2}}$ obey

$$
b^{-2} \frac{\partial^{2}}{\partial z^{2}}+\sum_{i=1}^{n-1}\left(\frac{\Delta_{i}}{\left(z-z_{i}\right)^{2}}+\frac{1}{\left(z-z_{i}\right)} \frac{\partial}{\partial z_{i}}\right)\left\langle V_{-\frac{b}{2}}(z) \prod_{i=1}^{n-1} V_{i}\left(z_{i}\right)\right\rangle=0
$$

Taking the (semi)classical limit $b \rightarrow 0$ in (2.11) ultimately recovers (2.5) as done around Section 2.1. Of course, a direct analogy can soon be seen in hermitian matrix models upon using the $c=1$ $\phi_{K S}(z)$; namely, the vev of a determinant operator

$$
\langle\operatorname{det}(z-M)\rangle=\left\langle\exp \left(\frac{1}{\hbar} \phi_{K S}\right)\right\rangle=\exp \left(\frac{1}{\hbar} \int^{z} d y\left\langle\partial \phi_{K S}(y)\right\rangle+\mathcal{O}\left(\hbar^{0}\right)\right)
$$

has been known to solve the Schrödinger-like equation stemming from the genus-zero spectral curve and meanwhile serves as the orthogonal polynomial for the matrix model. For example, (2.12) may stand for $H_{N}(z)$ (Hermite polynomial) when $Z$ is Gaussian.

\subsubsection{Reproducing Seiberg-Witten prepotential}

Let us proceed to work out some examples explicitly in which $2 N_{c}=N_{f}=4$ Seiberg-Witten prepotentials are recovered upon employing known classical 4pt-spheric conformal blocks. Extracting SW prepotentials from classical conformal blocks may be viewed as another derivation more or less independent of the original AGT proposal. This is because we have just taken advantage of both the Ward identity of the stress-tensor intrinsic to the uniformization problem (Polyakov conjecture) and arguments familiar in large- $N$ hermitian matrix models.

Here, bare flavor masses and weights assigned to punctures are related by

$$
\begin{cases}\xi_{1}=m_{1}+m_{2}+\frac{1}{2}, & \xi_{2}=-m_{1}+m_{2}+\frac{1}{2} \\ \xi_{3}=m_{3}+m_{4}+\frac{1}{2}, & \xi_{4}=-m_{3}+m_{4}+\frac{1}{2}\end{cases}
$$

which descends directly from the so-called AGT dictionary [1]. Besides, in [29] Teschner pointed out that (2.5) is referred to as the "Baxter equation" and applying to it the WKB approximation at the zeroth-order (much resembling (2.12) ) gives

$$
a \equiv \oint d z \sqrt{T(z)}=\frac{\ell\left(\gamma_{12}\right)}{4 \pi b} \quad \text { or } \quad p_{s}=\frac{a}{b} .
$$

Notice that $a$ expressed in terms of a period integral of $\sqrt{T(z)}$ coincides with the $S U(2)$ Coulomb phase parameter as claimed in [9, 1]. Equipped with these, we first examine the case of four massless flavors. 
(I) All $m_{i}=0$

In view of (2.13), under the large- $p_{s}$ limit implemented by $b \rightarrow 0, f_{\delta_{s}, \delta_{i}}(x)\left(\forall \delta_{i}=\frac{1}{4}\right)$ one has

$$
\begin{aligned}
& f_{\delta_{s}, \delta_{i}=\frac{1}{4}}(x) \\
& =\left(p_{s}^{2}-\frac{1}{4}\right) \log x+\left(p_{s}^{2}+\frac{1}{4}\right) \frac{x}{2}+\left(\frac{13 p_{s}^{2}}{16}+\frac{9}{32}+\frac{1}{256 p_{s}^{2}+256}\right) \frac{x^{2}}{4}+\cdots \\
& \rightarrow \frac{a^{2}}{b^{2}}\left(\log x+\frac{x}{2}+\frac{13 x^{2}}{64}+\frac{23 x^{3}}{192}+\cdots\right) \equiv \frac{1}{b^{2}} \mathcal{F}_{\text {inst }}^{S W}
\end{aligned}
$$

where in the second line we have borrowed (8.20) of Zamolodchikov and Zamolodchikov [11. Notice that the last line is precisely the desired SW prepotential (up to a perturbative piece $-\log 16$ ) via $b \equiv \hbar$ as well as $x \equiv \exp \left(2 \pi i \tau_{U V}\right)$.

(II) $m_{1}=m_{2}=\frac{\xi}{4}$ and $m_{3}=m_{4}=0$

Let us quote from [8] the following classical conformal block

$$
\begin{aligned}
f_{\frac{1}{4}+p^{2}}\left[\begin{array}{cc}
\frac{1}{4} & \frac{1-\xi^{2}}{4} \\
\frac{1}{4} & \frac{1}{4}
\end{array}\right](x) \quad & =\left(p^{2}-\frac{1-\xi^{2}}{4}\right) \log x+\left(\frac{1-\xi^{2}}{8}+\frac{p^{2}}{2}\right) x \\
& +\left(\frac{9\left(1-\xi^{2}\right)}{128}+\frac{13 p^{2}}{64}+\frac{\left(1-\xi^{2}\right)^{2}}{1024\left(1+p^{2}\right)}\right) x^{2}+\mathcal{O}\left(x^{3}\right) .
\end{aligned}
$$

Again, by $b \rightarrow 0$ which leads to both large- $p$ and large- $\xi$ limits, we finally observe that (2.15) does correctly reproduce the instanton part of SW prepotential for $\vec{m}=(m, m, 0,0)$ [30]:

$$
\mathcal{F}_{\text {inst }}^{S W}=\left(a^{2}-m^{2}\right) \log x+\left(a^{2}+m^{2}\right) \frac{x}{2}+\left(13 a^{2}+18 m^{2}+\frac{m^{4}}{a^{2}}+\mathcal{O}\left(a^{-4}\right)\right) \frac{x^{2}}{64}+\mathcal{O}\left(x^{3}\right)
$$

through carrying out $\left(a^{2}, m^{2}\right) \rightarrow\left(p^{2},-\frac{\xi^{2}}{4}\right)$.

\section{(III) Arbitrary four flavor masses}

In order to deal with this case, we quote from [1] the asymptotic expansion for generic $\delta_{i}$ and take both large $p$ - and $\xi_{i}$-limit, i.e.

$$
\begin{aligned}
f_{\delta, \delta_{i}}(x) \quad & =\left(\delta-\delta_{1}-\delta_{2}\right) \log x+\frac{\left(\delta+\delta_{1}-\delta_{2}\right)\left(\delta+\delta_{3}-\delta_{4}\right)}{2 \delta} x+\mathcal{O}\left(x^{2}\right) \\
& \rightarrow\left(a^{2}+2 m_{1}^{2}+2 m_{2}^{2}\right) \log x+\frac{a^{4}-4 a^{2}\left(m_{1} m_{2}+m_{3} m_{4}\right)+16 m_{1} m_{2} m_{3} m_{4}}{2 a^{2}} x+\cdots
\end{aligned}
$$

In the second line by $m_{k} \rightarrow \frac{i}{2} m_{k}$ one recovers the instanton part of $S U(2)$ (instead of $\left.U(2)\right)$ SW prepotential. For instance, it becomes

$$
\left(a^{2}-m^{2}\right) \log x+\frac{\left(a^{4}+2 a^{2} m^{2}+m^{4}\right)}{2 a^{2}} x+\cdots
$$

for $m_{1}=m_{2}=m_{3}=m_{4}=\frac{i}{2} m$ which agrees to (3.48) of [24] up to the $U(1)$ part $-2\left(m_{1}+\right.$ $\left.m_{2}\right)\left(m_{3}+m_{4}\right) \log (1-x)$. Certainly, in order to get higher-order corrections one finally has to 
appeal to the BPZ representation of conformal blocks:

$$
\begin{aligned}
& \mathcal{F}_{\Delta}\left(\Delta_{1,2,3,4} ; x\right)=x^{\frac{Q^{2}}{4}-\Delta_{1}-\Delta_{2}}(1-x)^{\frac{Q^{2}}{4}-\Delta_{1}-\Delta_{3}} \\
& \times(16 q)^{P^{2}}\left(\theta_{3}(q)\right)^{3 Q^{2}-4\left(\Delta_{1}+\Delta_{2}+\Delta_{3}+\Delta_{4}\right)} H_{\Delta}\left(\Delta_{i} ; q\right)
\end{aligned}
$$

where $x$ and $q$ are related by the celebrated elliptic lambda-function:

$$
x=\frac{\theta_{2}^{4}(q)}{\theta_{3}^{4}(q)}=16 q-128 q^{2}+704 q^{3}-3072 q^{4}+11488 q^{5}+\cdots
$$

with $q=\exp (i \pi \tau)$ and $\tau$ being called half-period. By first executing Zamolodchikov's recursive formula [31] for $H=1+\sum_{n=1}^{\infty} H_{(n)} q^{n}$ and then taking $\left(\alpha_{i}, P\right) \gg Q$ inside (2.17) (without the intermediate $b \rightarrow 0$ procedure) one is able to obtain to any desired order the $2 N_{c}=N_{f}=4 \mathrm{SW}$ prepotential.

\section{Application}

We have learned that dealing with uniformizing $C_{g, n}$ gets equivalent to studying Fuchsian equations (1.1) whose solutions involve conformal blocks within which at least one $\Phi_{2,1}$ is inserted. A contact with well-known integrable systems (Heun, Sutherland, etc.) made by (1.1) hopefully lays down opportunities for gaining insights into the corresponding $\mathcal{N}=2^{*} S U(2) \operatorname{SCFT}$ and $\mathcal{T}_{0,3}\left(A_{1}\right)$, a theory of four free hypermultiplets.

First of all, the solution to Heun equations must be interpreted as a spheric five-point conformal block $\mathcal{B}_{5} 8$ under $b \rightarrow 0$. Subsequently, by using Heun/eCM duality this $\mathcal{B}_{5}$ coincides with a toric $\mathcal{B}_{2}$ which as well as $\mathcal{B}_{5}$ involves one $\Phi_{2,1}$ insertion. Thereafter, we consider a limit taking eCM to Sutherland model which corresponds to the operation of pinching a torus at one point. Additionally, its eigenstates are examined by making use of toric diagrams engineering responsible $\mathcal{T}_{0,3}\left(A_{1}\right)$ systems.

\subsection{Calogero-Moser/Heun duality}

We quickly review Calogero-Moser/Heun duality. Recall that Heun equation is of second-order:

$$
\left(\left(\frac{d}{d z}\right)^{2}+\left(\frac{\gamma}{z}+\frac{\delta}{z-1}+\frac{\epsilon}{z-t}\right) \frac{d}{d z}+\frac{\alpha \beta z-q}{z(z-1)(z-t)}\right) F(z)=0
$$

with the constraint $\gamma+\delta+\epsilon=\alpha+\beta+1$. This differential equation is of Fuchsian type, namely, all singularities $(0,1, t, \infty)$ have to be regular.

It should be emphasized that any second-order differential equation with four regular singularities on $\mathbb{P}^{1}$ falls into Heun upon suitable transformations. Due to

$$
\wp(x)=\wp\left(x \mid 2 \omega_{1}, 2 \omega_{3}\right)=\frac{1}{x^{2}}+\sum_{(m, n) \in \mathbb{Z} \times \mathbb{Z} \backslash(0,0)}\left(\frac{1}{\left(x-2 m \omega_{1}-2 n \omega_{3}\right)^{2}}-\frac{1}{\left(2 m \omega_{1}+2 n \omega_{3}\right)^{2}}\right),
$$

the fundamental period of Weierstrass function falls into $\left(2 \omega_{1}, 2 \omega_{3}\right)$, i.e. $\wp\left(x+2 \omega_{1}\right)=\wp\left(x+2 \omega_{3}\right)=$ $\wp(x)$. A torus $\mathbb{C} /\left(2 \omega_{1} \mathbb{Z}+2 \omega_{3} \mathbb{Z}\right)$ thus comes from $\mathbb{P}^{1}$ owning branching points $\left(\omega_{0}, \omega_{1}, \omega_{2}, \omega_{3}\right)$ with $\omega_{0}=0$ and $\omega_{2}=-\omega_{1}-\omega_{3}$.

\footnotetext{
${ }^{8} \mathcal{B}_{n}$ is an abbreviation for an $n$-point conformal block.
} 
Also, by setting

$$
e_{i}=\wp\left(\omega_{i}\right), \quad t=\frac{e_{3}-e_{1}}{e_{2}-e_{1}}, \quad z=\frac{\wp(x)-e_{1}}{e_{2}-e_{1}}, \quad(i=1,2,3)
$$

four branching points are brought to $(0,1, t, \infty)$. Moreover, through $\eta(z)=z^{-\frac{l_{0}}{2}}(z-1)^{-\frac{l_{1}}{2}}(z-t)^{-\frac{l_{2}}{2}}$ and $F(z) \eta(z)=f(x)$ Heun equation is transformed into

$$
\left(-\frac{d^{2}}{d x^{2}}+\sum_{i=0}^{3} l_{i}\left(l_{i}+1\right) \wp\left(x+\omega_{i}\right)-E\right) f(x)=0
$$

where parameters are related by

$$
\begin{aligned}
& l_{0}=\beta-\alpha-1 / 2, \quad l_{1}=-\gamma+1 / 2, \quad l_{2}=-\delta+1 / 2, \quad l_{3}=-\epsilon+1 / 2, \\
& E=\left(e_{2}-e_{1}\right)\left(-4 q+\left(-(\alpha-\beta)^{2}+2 \gamma^{2}+6 \gamma \epsilon+2 \epsilon^{2}-4 \gamma-4 \epsilon-\delta^{2}+2 \delta+1\right) / 3\right. \\
& \left.+\left(-(\alpha-\beta)^{2}+2 \gamma^{2}+6 \gamma \delta+2 \delta^{2}-4 \gamma-4 \delta-\epsilon^{2}+2 \epsilon+1\right) t / 3\right) .
\end{aligned}
$$

Note that transforming (3.1) into (3.2) is generally not unique. When $l_{1}=l_{2}=l_{3}=0(\gamma=\delta=$ $\epsilon=1 / 2)$, (3.2) is called Lamé equation.

\subsection{Sphere versus torus}

Quite analogous to hypergeometric differential equations, Heun ones are designated to deal with four regular singularities placed on $\mathbb{P}^{1}$. Based on previous discussions, $\lim _{b \rightarrow 0} \mathcal{B}_{5}$ having one $\Phi_{2,1}$ insertion is naturally supposed to obey Heun equations. We will show that this is true upon using the result of Fateev, Litvinov, Neveu and Onofri [32] together with Heun/eCM duality.

In 32 B $\mathcal{B}_{5}$ was written down as 9

$$
\begin{aligned}
& \mathcal{B}_{5} \equiv\left\langle V_{-\frac{b}{2}}(z) V_{1}(0) V_{2}(1) V_{3}(\infty) V_{4}(x)\right\rangle=z^{\frac{1}{2 b^{2}}}(z-1)^{\frac{1}{2 b^{2}}} \\
& \times \frac{(z(z-1)(z-x))^{\frac{1}{4}}}{(x(x-1))^{\frac{8 \Delta_{4}+1}{12}}} \frac{\Theta_{1}(u)^{\frac{1}{b^{2}}}}{\Theta_{1}^{\prime}(0)^{\frac{1+b^{2}}{3 b^{2}}}} \Phi(u \mid \tau)
\end{aligned}
$$

where

$$
\begin{array}{ll}
\left(-\partial_{u}^{2}+V(u)\right) \Phi(u \mid \tau)=\frac{4 i}{\pi b^{2}} \partial_{\tau} \Phi(u \mid \tau), & V(u)=\sum_{i=0}^{3} s_{i}\left(s_{i}+1\right) \wp\left(u-\omega_{i}\right), \\
u=\frac{\pi}{4 K(x)} \int_{0}^{(z-x) /(x z-x)} \frac{d t}{\sqrt{t(1-t)(1-x t)}}, & 2 \alpha_{i}=Q-b\left(s_{i}+\frac{1}{2}\right) .
\end{array}
$$

Notice that $\Theta_{1}(u)$ is Jacobi theta function whilst $K(x)$ denotes the complete elliptic integral of the first kind. By $b \rightarrow 0$ one observes that

$$
\begin{aligned}
& \left(-b^{2} \partial_{u}^{2}+U(u)\right) \Phi^{c l}(u \mid \tau)=\frac{4 i}{\pi} \partial_{\tau} \Phi^{c l}(u \mid \tau), \\
& U(u)=\sum_{i=0}^{3} \ell_{i}\left(\ell_{i}+b\right) \wp\left(u-\omega_{i}\right), \quad \ell_{i}=\left(\frac{1}{b}-2 \alpha_{i}\right) .
\end{aligned}
$$

\footnotetext{
${ }^{9}$ For the sake of brevity, $V_{i}$ stands for $V_{\alpha_{i}}$.
} 
When $\ell_{i}=b l_{i}$, via the transformation technique advocated within (3.1)-(3.3), one realizes that up to a prefactor $\lim _{b \rightarrow 0} \mathcal{B}_{5} \sim \Phi^{c l}(u \mid \tau)$ does obey Heun equation.

Remarkably, in [33, 34] it was shown that toric two-point conformal blocks under $b \rightarrow 0$ satisfies Lamé $\left(s_{1}=s_{2}=s_{3}=0\right)$ equation to which Heun can reduce, i.e.

$$
\begin{aligned}
& \left\langle V_{-\frac{b}{2}}(z) V_{\alpha}(0)\right\rangle_{\tau}=\Theta_{1}(z)^{\frac{b^{2}}{2}} \eta(\tau)^{2 \Delta_{\alpha}-1-2 b^{2}} \Psi(z \mid \tau), \\
& \left(-b^{-2} \partial_{z}^{2}+\alpha^{2} \wp(z)\right) \Psi^{c l}(z \mid \tau)=\frac{2 i}{\pi} \partial_{\tau} \Psi^{c l}(z \mid \tau), \quad b \rightarrow 0, \quad b \alpha: \text { finite. }
\end{aligned}
$$

Upon performing the WKB method onto these Schrödinger-like equations, one is capable of conjecturing the equivalence between spheric four-point and toric one-point BPZ conformal blocks (without $\Phi_{2,1}$ ) under special momentum assignments just as argued around (3.29) in [32] (see also [35, 36]). Certainly, along the line of AGT conjecture, relating these pure Liouville stuffs to elliptic/non-elliptic $\mathcal{N}=2 S U(2) \times S U(2)$ SCFTs and studying their Nekrasov instanton partition functions with surface operators inserted under NS limit analogous to [16] remain good future issues.

\subsection{Sutherland model}

Let us pause momentarily to consider how eCM can be brought to Sutherland (or trigonometric Calogero-Sutherland) model. In fact, this is quite straightforward, i.e. given eCM Hamiltonian

$$
H_{E} \equiv \frac{1}{4 \pi^{2}}\left(-\sum_{i=1}^{N} \frac{\partial^{2}}{\partial x_{i}^{2}}+2 l(l+1) \sum_{1 \leq i<j \leq N} \wp\left(x_{i}-x_{j}\right)\right)
$$

where $l$ is the coupling constant and the fundamental periodicity of Weierstrass $\wp$-function is $(1, \tau)$, via $\tau \rightarrow i \infty$ or $q=\exp (\pi i \tau) \rightarrow 0$ one obtains from $H_{E}$

$$
H_{S} \equiv \frac{1}{2}\left(-\sum_{i=1}^{N} \frac{\partial^{2}}{\partial x_{i}^{2}}+\sum_{1 \leq i<j \leq N} \frac{2 \beta(\beta-1) \pi^{2}}{\sin ^{2} \pi\left(x_{i}-x_{j}\right)}\right) .
$$

In order to compute wave functions of $H_{S}$ we redefine it by means of the ground state $\Delta(X)$ $\left(X_{i}=e^{2 \pi i x_{i}}\right)$ as

$$
H_{0} \equiv \Delta(X)^{-1}\left(H_{S}-e_{0}\right) \Delta(X), \quad \Delta(X) \equiv\left(\frac{1}{\pi} \prod_{i<j} \sin \pi\left(x_{i}-x_{j}\right)\right)^{\beta}
$$

where $e_{0}$ comes from $H_{S} \Delta(X)=e_{0} \Delta(X)$ and $\Delta(X)$ reduces to simply an unitary Vandermonde at $\beta=1$. Now, a well-known fact is that $H_{0}$ owns symmetric Jack polynomials as its excited eigenstates $(\lambda$ : Young tableaux)

$$
H_{0} J_{\lambda}(X)=E_{\lambda} J_{\lambda}(X)
$$

which reduce to Schur polynomials at $\beta=1$. As pointed out in [37, a collective chiral boson description responsible for $J_{\lambda}(X)$ exists and its Virasoro central charge is related to $\beta$ by $c=$ $1-\frac{6(1-\beta)^{2}}{\beta}$. We will return to this aspect soon. 


\subsection{Gegenbauer versus $\mathcal{T}_{0,3}\left(A_{1}\right)$}

While a gauge-transformed $H_{S}$ in (3.7) gets reduced to a two-body $A_{1}$-system, it is widely known that its eigenstates become Gegenbauer polynomials:

$$
C_{n}^{\nu}(x)=\frac{\Gamma(n+2 \nu)}{\Gamma(2 \nu) n !} \cdot{ }_{2} F_{1}\left(-n, n+2 \nu, \nu+\frac{1}{2} ; \frac{1-X}{2}\right) .
$$

Though $C_{n}^{\nu}(x)$ has to be viewed as some $\lim _{b \rightarrow 0} \mathcal{B}$ as stressed, it nevertheless can stand for a $b=i$ (4D physical limit) solution to (3.4) being exact w.r.t. $b$ whenever $\beta$ in (3.7) gets necessarily renormalized to, say, $\tilde{\beta}$. This special value of $b$ will amount to facilitating our comparison with "unrefined" information encoded in ordinary toric diagrams.

In the presence of ${ }_{2} F_{1}(\cdots)$ in (3.9), we legally doubt whether it has something to do with the spheric $\mathcal{B}_{4}(b=i)$ having one $\Phi_{2,1}$ insertion. Usually, $\left\langle V_{-\frac{b}{2}}(x) V_{1}(0) V_{2}(1) V_{3}(\infty)\right\rangle \equiv{ }_{2} F_{1}(A, B, C ; x)$ where

$$
\left\{\begin{array}{l}
A=-N \\
B=\frac{1}{\beta}\left(-\frac{2 \alpha_{1}}{\epsilon_{1}}-\frac{2 \alpha_{2}}{\epsilon_{1}}+2\right)+N-1, \\
C=\frac{1}{\beta}\left(-\frac{2 \alpha_{1}}{\epsilon_{1}}+1\right), \\
N=-\epsilon_{1}\left(\alpha_{1}+\alpha_{2}-\alpha_{3}-\frac{b}{2}\right), \quad \beta=-\frac{\epsilon_{2}}{\epsilon_{1}}, \quad \epsilon_{1}=b, \quad \epsilon_{2}=\frac{1}{b}
\end{array}\right.
$$

Henceforth, that arguments inside ${ }_{2} F_{1}(\cdots ; x)$ of $C_{n}^{\nu}(x)$ are not all independent tells us that only two of three $\alpha_{i}$ 's are independent. Still, note that $X=\exp (2 \pi i x) \in \mathbb{C}^{*}$ makes the periodicity of $x \in \mathbb{C}$ even explicit. Let us render a justification in the following paragraph.

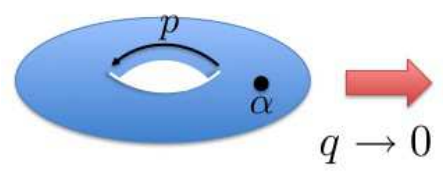

Calogero-Moser model
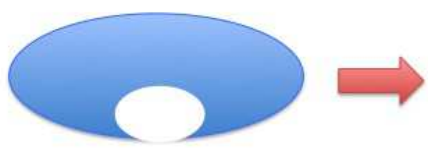

Sutherland model

Fig. 1: Carrying out the limit $q \rightarrow 0$ brings one from Calogero-Moser to Sutherland. Note that weights of three punctures are not independent because only two parameters $(\alpha, p)$ (converted into $\mathcal{N}=2^{*} S U(2)$ Coulomb branch parameter a and adjoint hypermultiplet mass $\mathbf{m}$ via AGT dictionary) are present.

As shown around (3.5), we manage to think of (3.9) as a toric $\mathcal{B}_{2}(b=i)$ having one $\Phi_{2,1}$ insertion defined on a pinched torus as explained in Figure 1, On the other hand, by adhering to [1, 9], this kind of Riemann surface can also correspond to the physical $4 \mathrm{D} \mathcal{T}_{0,3}\left(A_{1}\right)$ theory of four free hypermultiplets whose bare masses are evaluated by $\alpha_{1} \pm \alpha_{2} \pm \alpha_{3}$. However, because this $\mathcal{T}_{0,3}^{\prime}\left(A_{1}\right)$ is yielded via a degenerate limit of $\mathcal{T}_{1,1}\left(A_{1}\right)$ (that is why a prime is added onto $\mathcal{T}$ ) there must exist certain constraint between $\alpha_{i}$ 's. Combined with (3.9) and (3.10), one easily arrives at $\alpha_{1}=\alpha_{2}$. Let us see whether this is consistent with what is read off from a toric diagram associated with a Calabi-Yau three-fold engineering the physical $\mathcal{N}=2^{*} S U(2)$ theory at $\epsilon_{1}+\epsilon_{2}=0$ (or $b=i$ ). Based on Figure 2, one is able to measure masses of four hypermultiplets $m_{i}(i=1,2,3,4)$ in terms of the distance (or area) of blown-up parts of the RHS web diagram (b). Note also that the correspondence between (b) and $\mathcal{T}_{0,3}^{\prime}\left(A_{1}\right)$ (under the name $\tilde{T}_{2}$ strip) has been beautifully established in [15]. In 


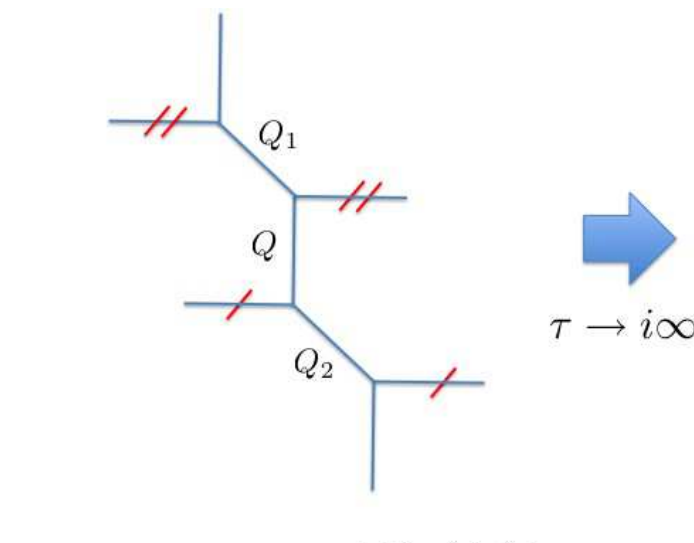

Calogero-Moser model $\left(\mathcal{T}_{0,3}\left(A_{1}\right)\right)$

(a)

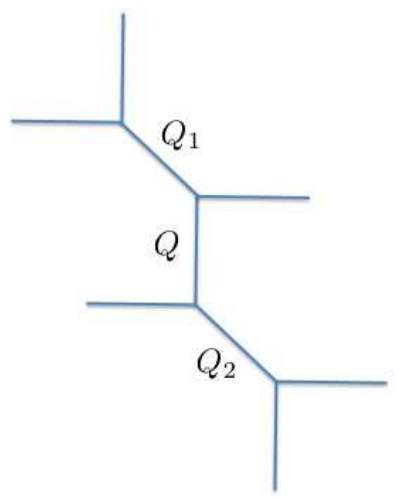

Sutherland model $\left(\mathcal{T}_{0,3}^{\prime}\left(A_{1}\right)\right)$

(b)

Fig. 2: Transition from $\mathcal{T}_{0,3}\left(A_{1}\right)$ to $\mathcal{T}_{0,3}^{\prime}\left(A_{1}\right)$ through $\tau \rightarrow i \infty$.

general, these areas are basically five-dimensional, i.e. $Q_{i}=\exp \left(-R \mathbf{m}_{i}\right)$ and $Q=\exp (-R \mathbf{a})$ which fall into four-dimensional quantities when $R \rightarrow 0$ ( $R$ : size of M-circle). Accompanied by some necessarily constant shifts, one finds finally that $\left(m_{1}, m_{2}, m_{3}, m_{4}\right) \equiv \alpha_{1} \pm \alpha_{2} \pm \alpha_{3}$ subject to $\alpha_{1}=\alpha_{2}$ is fulfilled in Figure 2 due to $Q_{1}=Q_{2}\left(\mathbf{m}_{1}=\mathbf{m}_{2}\right)$ in $\mathcal{N}=2^{*}$ cases.

\subsection{Bubbling pants}

Symmetric Jack polynomials with multiple variables show up, given the following $k+3$ point Liouville conformal block [15, 38]:

$$
\frac{\left\langle\alpha_{1}\left|V_{2}(1) \prod_{i=1}^{k} V_{-\frac{b}{2}}\left(z_{i}\right)\right| \alpha_{3}+k \frac{b}{2}\right\rangle}{\left\langle\alpha_{1}\left|V_{2}(1)\right| \alpha_{3}+k \frac{b}{2}\right\rangle}
$$

which up to a prefactor $\prod_{i=1}^{k} z_{i}^{b \alpha_{1}}\left(1-z_{i}\right)^{b \alpha_{2}}$ is expressed in terms of a generalized hypergeometric function $(\gamma>0)$ :

$$
{ }_{p} F_{q}^{(\gamma)}\left(a_{1}, \cdots, a_{p} ; b_{1}, \cdots, b_{q} ; z_{1}, \cdots, z_{k}\right)=\sum_{\kappa} \frac{1}{|\xi| !} \cdot \frac{\left(a_{1}\right)_{\kappa}^{(\gamma)} \cdots\left(a_{p}\right)_{\kappa}^{(\gamma)}}{\left(b_{1}\right)_{\kappa}^{(\gamma)} \cdots\left(b_{q}\right)_{\kappa}^{(\gamma)}} \cdot C_{\kappa}^{(\gamma)}\left(z_{1}, \cdots, z_{k}\right)
$$

with $(p, q)=(2,1)$ and

$$
a_{1}=b\left(\alpha_{1}+\alpha_{2}-\alpha_{3}\right), \quad a_{2}=b\left(\alpha_{1}+\alpha_{2}+\alpha_{3}+Q\right), \quad b_{1}=2 b \alpha_{1} .
$$

Here, $\kappa$ denotes a random partition containing at most $k$ rows, $\left(a_{i}\right)_{\kappa}^{(\gamma)}$ the generalized Pochhammer symbol and $C_{\kappa}^{(\gamma)}\left(z_{1}, \cdots, z_{k}\right)$ the $C$-normalized Jack polynomial obeying

$$
\sum_{|\kappa|=\xi} C_{\kappa}^{(\gamma)}\left(z_{1}, \cdots, z_{k}\right)=\sigma_{1}\left(z_{i}\right)^{\kappa}, \quad \sigma_{n}\left(z_{i}\right)=\sum_{i_{1}<\cdots<i_{n}} z_{i_{1}} \cdots z_{i_{n}}
$$

Whenever we view $C_{\kappa}^{(\gamma)}\left(z_{1}, \cdots, z_{k}\right)$ as the eigenstate of $H_{0}$ in a $k$-body Sutherland model, $\gamma$ denotes some continuous parameter depending on $\beta$. Three momenta $\alpha_{i}$ 's at $b=i$ must also be properly 
constraint in a fashion mentioned before. Likewise, in a hermitian matrix model $Z$ for instance, when the tree-level potential of $Z$ is Gaussian ( $M: N \times N$ matrix) one has

$\mathcal{K}\left(z_{1}, \cdots, z_{k}\right) \equiv\left\langle\prod_{i=1}^{k} \operatorname{det}\left(z_{i}-M\right)\right\rangle \simeq \frac{\operatorname{det}\left(H_{N+j-1}\left(z_{i}\right)\right)}{\Delta(z)}, \quad H_{N}(z):$ orthogonal polynomial

where $\Delta(z)$ is the usual Vandermonde. Needless to say, $\mathcal{K}$ is an eigenstate of a $k$-body gaugetransformed Hamiltonian $H_{g}$

$$
H_{g}=\Delta(z)^{-1} H_{h} \Delta(z), \quad H_{h}=\sum_{i=1}^{k}-\frac{\partial^{2}}{\partial z_{i}^{2}}+z_{i}^{2} .
$$

Now we are in a position to ask what is the physical content of the situation: $\beta=1$ such that Jack reduces to Schur.

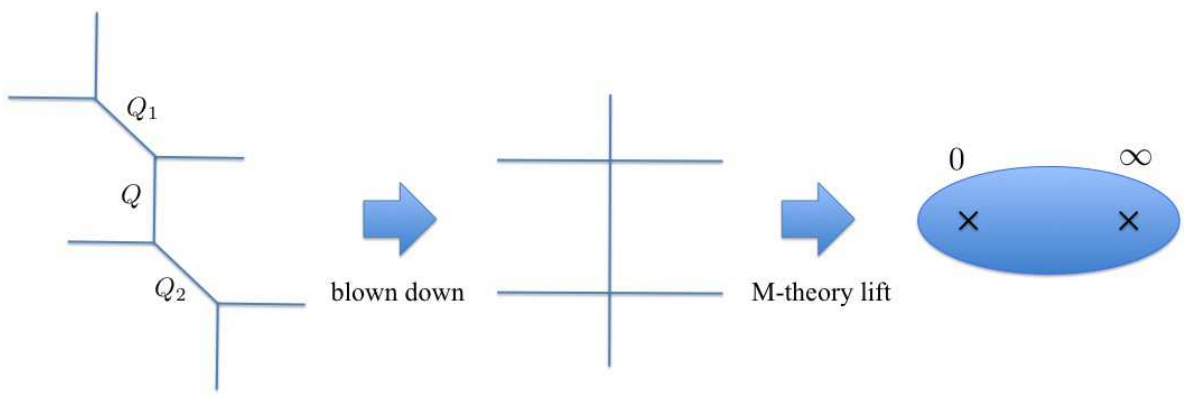

Fig. 3: A blown-down procedure leads to a singular toric diagram.

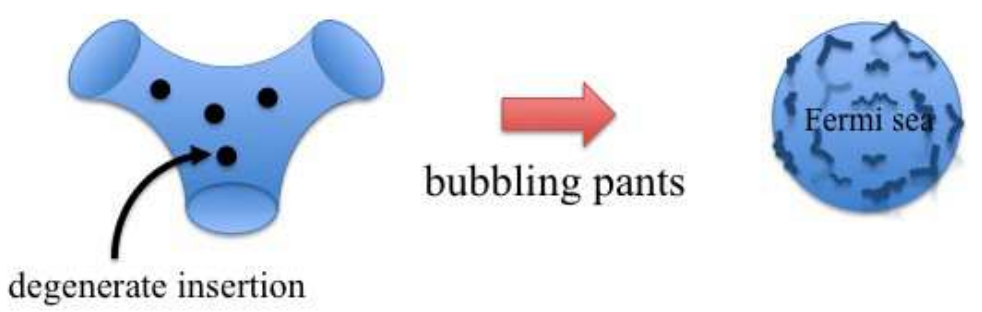

Fig. 4: Schematic picture of bubbling pants.

This time we try to resort to Figure 3 where $\left(Q_{1}, Q_{2}\right)$ are blown down to be zero-sized. This well echoes the fact that the critical value $\beta=1$ is just responsible for $\mathbf{m}=0$ (zero adjoint hypermultiplet mass) in view of (3.5) and (3.7). Moreover, in M-theory the central picture in Figure 3 is further lifted to a genus-zero Riemann surface punctured at $(0, \infty)$. More explicitly, one can think of this singular web diagram as what engineers $\mathcal{N}=2 N_{f}=2 N_{c}=2$ theory with zero flavor masses. Its SW curve to some crude extent gained by thickening the web diagram thereby looks like

$$
\omega+\frac{1}{\omega}=\frac{P_{1}(v)}{\sqrt{Q_{2}(v)}}
$$

representing a two-punctured Riemann sphere. Note that the subscript denotes the degree of a 
monic polynomial. Henceforth, that many degenerate $V_{-\frac{i}{2}}$ insertions at $\beta=1$ (RHS of Figure 3) are captured by Schur polynomials [37] now gets mapped to many tachyon excitations inserted on the asymptotic region of the self-dual $c=1$ Fermi liquid 10 . The reason is twofold. First, the asymptotic collective field describing the shape of $c=1$ Fermi surface is fermionized to precisely Schur polynomials defined by $\left(\lambda\right.$ : Young tableaux, $\lambda_{i}: i$-th row length) 11

$$
\langle\lambda \mid Z\rangle=\frac{\operatorname{det} z_{j}^{\lambda_{i}+k-i}}{\operatorname{det} z_{j}^{k-i}}, \quad i, j=1, \cdots, k .
$$

Second, the undisturbed $c=1$ Fermi liquid in the phase space is eventually mirrored to a twopunctured sphere as asserted in [41] via the topological B-model language. Now we have obtained from "bubbling pants" picture (Figure 4) the celebrated free-fermionic (Schur) nature of $c=1$ non-critical string theory. Namely, without the massless limit of $\mathcal{T}_{0,3}^{\prime}\left(A_{1}\right)$ we might never discover a description of the old $c=1$ story in terms of Sutherland model.

\section{Summary}

Let us summarize the main idea we are after in this article by a chart attached below.

\section{uniformization}

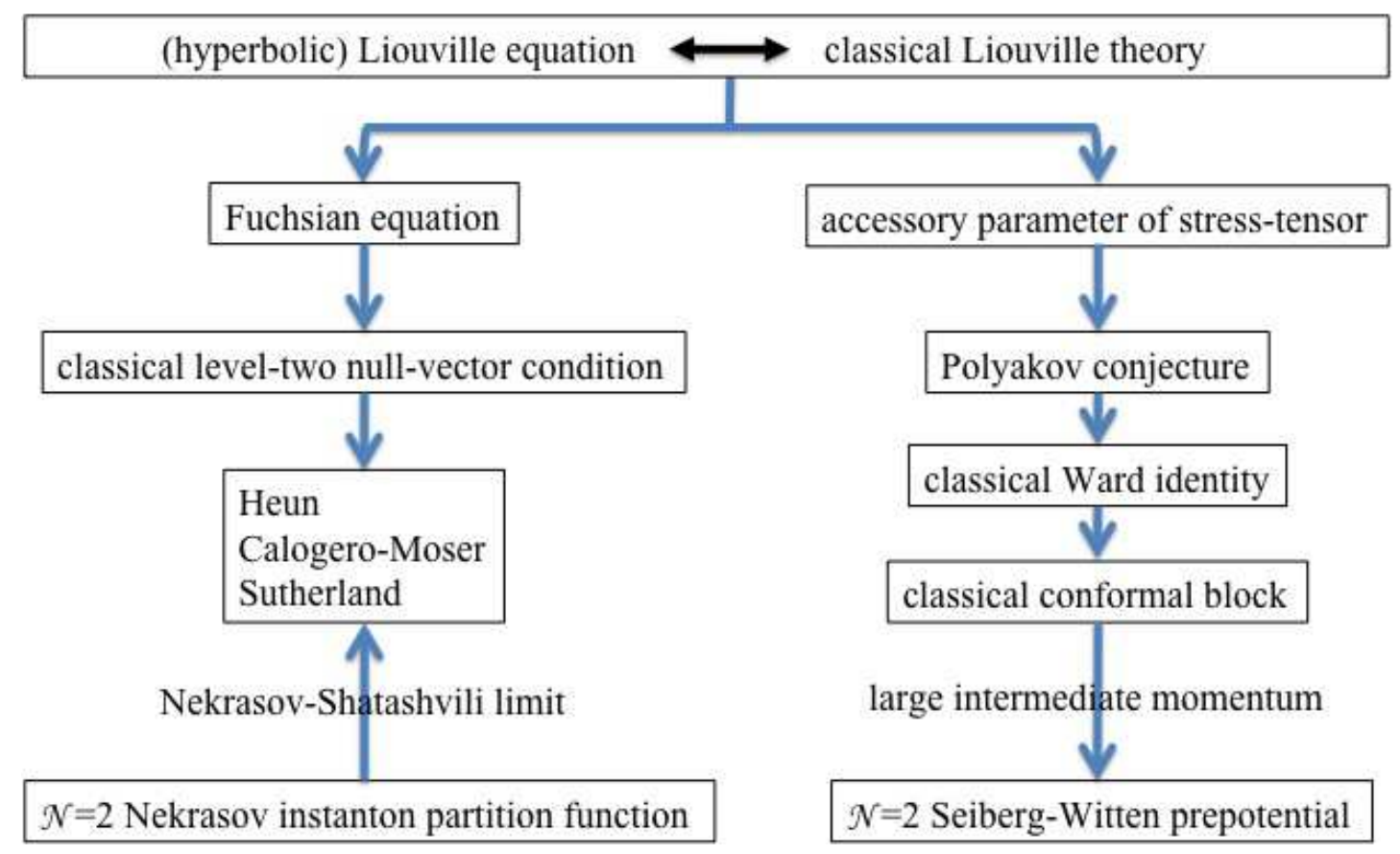

\footnotetext{
${ }^{10}$ For the sake of brevity, we will omit the word "self-dual".

${ }^{11}$ See [39, 40] for detailed discussions about $c=1$ string theory, Imbimbo-Mukhi type matrix model and Schur polynomials. In particular, in [39] $\mathcal{N}=4$ half-BPS correlators independent of Yang-Mills coupling constant $g_{Y M}$ are shown to coincide with $c=1$ tachyon scattering amplitudes to all genera. This fact may strengthen that the blown-down $\mathcal{T}_{0,3}^{\prime}\left(A_{1}\right)$ corresponds to $\mathcal{N}=4$ Yang-Mills with $g_{Y M} \rightarrow 0$.
} 


\section{Acknowledgements}

TST thanks Takeo Inami, Hirotaka Irie, Tetsuji Kimura, Kazuyoshi Maruyoshi, Yutaka Matsuo, Akitsugu Miwa, Shotaro Shiba, Masato Taki and Akihiro Tsuchiya for helpful communications. He owes very much Hideaki Iida for his discussions and numerous graphical supports. He is also grateful to organizers and participants of "3rd Mini Workshop on String Theory" held at KEK. TST is supported in part by the postdoctoral program at RIKEN.

\section{References}

[1] L. F. Alday, D. Gaiotto and Y. Tachikawa, Lett. Math. Phys. 91 (2010) 167 arXiv:0906.3219 [hep-th]].

[2] N. Wyllard, JHEP 0911 (2009) 002 arXiv:0907.2189 [hep-th]].

[3] D. Gaiotto, arXiv:0908.0307 [hep-th].

[4] A. Marshakov, A. Mironov and A. Morozov, Phys. Lett. B 682 (2009) 125 arXiv:0909.2052 [hep-th]].

[5] H. Awata and Y. Yamada, JHEP 1001 (2010) 125 [arXiv:0910.4431 [hep-th]].

[6] H. Awata and Y. Yamada, arXiv:1004.5122 [hep-th].

[7] L. Hadasz, Z. Jaskolski and M. Piatek, Nucl. Phys. B 724 (2005) 529 [arXiv:hep-th/0504204].

[8] L. Hadasz and Z. Jaskolski, J. Math. Phys. 47 (2006) 082304 arXiv:hep-th/0604187.

[9] D. Gaiotto, arXiv:0904.2715 [hep-th].

[10] N. A. Nekrasov and S. L. Shatashvili, arXiv:0908.4052 [hep-th].

[11] A. B. Zamolodchikov and A. B. Zamolodchikov, Nucl. Phys. B 477 (1996) 577 arXiv:hep-th/9506136.

[12] A. A. Belavin, A. M. Polyakov, and A. B. Zamolodchikov, "Infinite conformal symmetry in two-dimensional quantum field theory," Nucl. Phys. B 241 (1984) 333.

[13] L. F. Alday, D. Gaiotto, S. Gukov, Y. Tachikawa and H. Verlinde, JHEP 1001 (2010) 113 arXiv:0909.0945 [hep-th]].

[14] A. Mironov and A. Morozov, JHEP 1004 (2010) 040 [arXiv:0910.5670 [hep-th]].

[15] C. Kozcaz, S. Pasquetti and N. Wyllard, arXiv:1004.2025 [hep-th].

[16] L. F. Alday and Y. Tachikawa, arXiv:1005.4469 [hep-th].

[17] T. Dimofte, S. Gukov and L. Hollands, arXiv:1006.0977 [hep-th].

[18] K. Maruyoshi and M. Taki, arXiv:1006.4505 [hep-th].

[19] M. Taki, arXiv:1007.2524 [hep-th].

[20] H. Awata, H. Fuji, H. Kanno, M. Manabe and Y. Yamada, arXiv:1008.0574 [hep-th].

[21] C. Kozcaz, S. Pasquetti, F. Passerini and N. Wyllard, arXiv:1008.1412 [hep-th].

[22] T. S. Tai, arXiv:1006.0471 [hep-th].

[23] P. G. Zograf and L. A. Takhtajan, "On Liouville equation, accessory parameters and the geometry of Teichmüller space for Riemann surface of genus 0," Math. USSR Sbornik 60 (1988) 143. P. G. Zograf and L. A. Takhtajan, "Hyperbolic 2-spheres with conical singularities, accessory parameters and Kähler meitrics on $M_{0, n}$," Trans. Amer. Math. Soc. 355 (2003), 1857-1867 arXiv:math/0112170]. L. A. Takhtajan and L.-P. Teo, "Liouville action and Weil-Petersson metric on deformation spaces, global Kleinian reciprocity and holography," Commun. Math. Phys. 239 (2003), 183-240 arXiv:math/0204318. 
[24] T. Eguchi and K. Maruyoshi, JHEP 1007 (2010) 081 [arXiv:1006.0828 [hep-th]].

[25] R. Dijkgraaf and C. Vafa, arXiv:0909.2453 [hep-th].

[26] H. Itoyama, K. Maruyoshi and T. Oota, Prog. Theor. Phys. 123 (2010) 957 arXiv:0911.4244 [hep-th]].

[27] T. Eguchi and K. Maruyoshi, JHEP 1002 (2010) 022 [arXiv:0911.4797 [hep-th]].

[28] M. Fujita, Y. Hatsuda and T. S. Tai, JHEP 1003 (2010) 046 [arXiv:0912.2988 [hep-th]].

[29] J. Teschner, arXiv:1005.2846 [hep-th].

[30] A. Marshakov, A. Mironov and A. Morozov, JHEP 0911 (2009) 048 arXiv:0909.3338 [hepth]].

[31] Al. B. Zamolodchikov, Conformal symmetry in two-dimensional space: Recursion representation of conformal block, Theor. Math. Phys. 73 (1987) 1088.

[32] V. A. Fateev, A. V. Litvinov, A. Neveu and E. Onofri, J. Phys. A 42 (2009) 304011 arXiv:0902.1331 [hep-th]].

[33] T. Eguchi and H. Ooguri, Nucl. Phys. B 282 (1987) 308.

[34] V. A. Fateev and A. V. Litvinov, JHEP 1002 (2010) 014 [arXiv:0912.0504 [hep-th]].

[35] R. Poghossian, JHEP 0912 (2009) 038 [arXiv:0909.3412 [hep-th]].

[36] L. Hadasz, Z. Jaskolski and P. Suchanek, Phys. Lett. B 685 (2010) 79 arXiv:0911.4296 [hep-th]].

[37] H. Awata, Y. Matsuo, S. Odake and J. Shiraishi, Nucl. Phys. B 449 (1995) 347 arXiv:hep-th/9503043].

[38] J. Kaneko, "Selberg integrals and hypergeometric functions associated with Jack polynomials," SIAM J. Math. Anal. 4 (1993) 1086.

[39] T. S. Tai, Prog. Theor. Phys. 119 (2008) 165 arXiv:0709.0432 [hep-th]].

[40] T. S. Tai, Prog. Theor. Phys. 117 (2007) 795 arXiv:hep-th/0701086.

[41] M. Aganagic, R. Dijkgraaf, A. Klemm, M. Marino and C. Vafa, Commun. Math. Phys. 261 (2006) 451 arXiv:hep-th/0312085. 